\section{Decay Curves of Uranium and Thorium Fission Products}

IT has been suggested by Bohr ${ }^{1}$ that the rare isotope ${ }_{9.2}^{23.5} \mathrm{U}$ is responsible for the fission produced in uranium by slow neutrons, while the fission produced by fast neutrons is to be ascribed to the abundant isotope ${ }_{9.2}^{2: 38} \mathrm{U}$. In this connexion it is of interest to see whether any differences can be detected between the shapes of the resulting decay curves for the fission products of uranium bombarded with fast

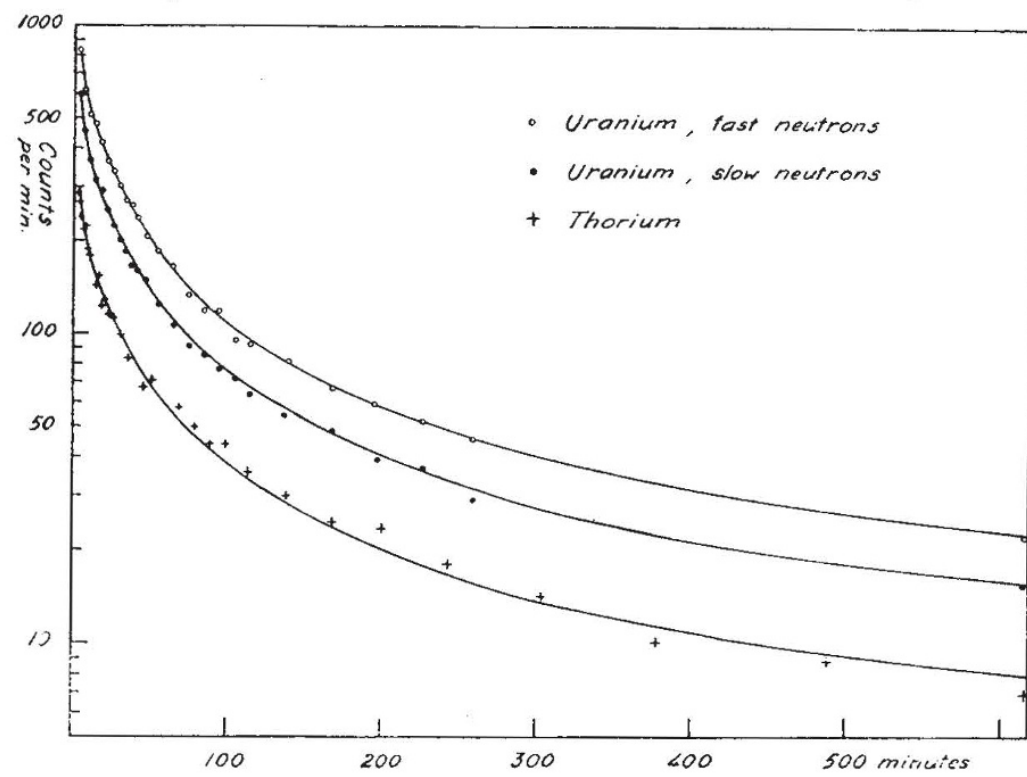

DECAY CURVES FOR THE DISINTEGRATION OF URANIUM BY FAST AND SLOW NEUTRONS, AND FOR THE DISINTEGRATION OF THORIUM

and slow neutrons, and also to compare these curves with those obtained with thorium. Some experiments by $\mathrm{F}$. Joliot ${ }^{2}$ indicated that slow neutrons were relatively more effective in producing the longer period activities than the shorter.

We have made similar experiments using neutrons from a lithium target bombarded with 50 microamperes of 750 kilovolt deuterons. The fission products were collected by recoil on a collector plate placed $1 \mathrm{~mm}$. below a glass plate painted with uranium or thorium oxide. In the fast neutron experiments, these plates were surrounded with cadmium sheets and placed immediately below the lithium target. In the slow neutron experiments a $4 \mathrm{~cm}$. slab of paraffin wax was interposed between the target and the plates, which were resting on another block of paraffin wax, $8 \mathrm{~cm}$. thick. In the latter case 75 per cont of the activity obtained was due to slow neutrons (only 25 per cent remaining when the plates were shielded with cadmium).

In three series of experiments, uranium was bombarded for 20 seconds, 3 minutes and 100 minutes, respectively, care being taken to keep the neutron intensity constant during the irradiation. In the first two cases the collector was of lead, while in the 100-minute experiment paper was used. Afterwards the activity of the collector plates was measured with a Geiger counter connected through a Neher-Harper circuit to a 'scale of eight', which had been tested for linear counting up to 5,000 particles per minute. The accompanying graph shows the decay curves obtained with 100 minutes exposure; each curve represents two series of measurements. The shape of the curve is the same for fast and for slow neutrons. This was also the caso in the experiments with 3 minutes exposure and 20 seconds exposure, where several series of measurements were taken and the decay was followed for 30 minutes and 5 minutes respectively. In no case did the ratio of the activity for fast and slow neutrons change during the decay; a change of 10 per cent would have been noticed.

As regards the thorium fission products, the shape of the decay curve is again indistinguishable from that of the uranium fission products for the same time of exposure. In order to avoid contamination with the active. deposit from thorium emanation, the thorium oxide layer was sealed up behind a celluloid foil of $0.4 \mathrm{~mm}$. air equivalent. In the ease of the 100 minutes exposure this was, however, not sufficient, and only when the emanation was continuously blown away during the exposure was this contamination avoided.

From the measurements just reported, it thus seems impossible to decide whether different uranium isotopes are responsible for the fission produced by slow and fast neutrons. Although some of the periods for the fission products from different nuclei must be different, the abundance of periods of any duration seems to be so great that these differences are smoothed out in the resulting decay curve.

We wish to thank Prof. Niels Bohr for suggesting the problem, and Prof. L. Meitner and Dr. O. R. Frisch for help and advice.

T. BJERGE.

K. J. Brostrom J. КоCH.

Institute of Theoretical Physics,

Cniversity of Copenhagen. May 2.

${ }^{2}$ Bohr, N., Phus. Rer., 55, 418 (1939).

${ }^{2}$ Joliot, F., J. Phis., 10, 159 (1939).

\section{Tracks on Photographic Plates of the Recoi Nuclei of Disintegration of Uranium}

UNDER the bombardment of uranium by neutrons, recoil nuclei of great mass and high ionizing power are produced. According to the measurements of Frisch ${ }^{1}$, the ionization which they produce along their tracks attains $70 \mathrm{Mev}$. Their range in air, measured by Joliot ${ }^{2}$, is nearly $3 \mathrm{~cm}$.

For these reasons, it was to be expected that the tracks of such recoil atoms would be observed on photographic plates with a thick coat of emulsion. Our first experiments ${ }^{3}$ suggested this assumption. On a plate, $2 \mathrm{~cm} . \times 3 \mathrm{~cm}$., some thick and short tracks were obtained, which can be attributed to the uranium recoil nuclei. A preparation of radiothorium plus beryllium being taken as a source of neutrons, besides the recoil tracks a considerable background of $\gamma$-rays was also observed on the plates. 